\title{
Necessidades humanas básicas dos profissionais de enfermagem: situações de (in)satisfação no trabalho*
}

\author{
BASIC HUMAN NEEDS OF NURSING PROFESSIONAL: SITUATIONS OF \\ (DIS)SATISFACTION AT WORK
}

NECESIDADES HUMANAS BÁSICAS DE LOS PROFESIONALES DE ENFERMERÍA:
SITUACIONES DE (IN)SATISFACCIÓN EN EL TRABAJO

\section{Lorena Fagundes Ladeia Vitoria Regis ${ }^{1}$, Isaura Setenta Porto ${ }^{2}$}

\section{RESUMO}

O cuidado está associado às ações da enfermagem considerando, sobretudo um conjunto de necessidades da equipe de enfermagem. Os objetivos desta pesquisa foram: caracterizar situações de (in)satisfações da equipe de enfermagem no trabalho e analisar as implicações destas (in)satisfações da equipe de enfermagem no trabalho. Adotouse a teoria das necessidades humanas básicas de Maslow para compreender fatores motivacionais e utilizou-se uma metodologia qualitativa com aplicação de técnicas quantitativas. O método compôs-se de observação participante com registros em diário de campo associada à aplicação de um questionário para 18 participantes da equipe de enfermagem em um Hospital Público do Rio de Janeiro. O estudo indica que as necessidades básicas da equipe de enfermagem encontram-se comprometidas, principalmente as necessidades de segurança e fisiológicas, as mais primárias. O comprometimento das necessidades primárias do sujeito que cuida implica em prejuízo para a saúde e redução do desempenho no trabaIho em cenários hospitalares.

\section{DESCRITORES}

Equipe de enfermagem Satisfação no emprego Saúde do trabalhador

\begin{abstract}
Care is associated with the nursing actions considering, above all, a group of needs of the nursing team. The objectives of this study were: to characterize situations of (dis) satisfaction of the nursing team at work and analyze their possible implications. Maslow's theory of basic human needs was used to understand the motivational factors, and a qualitative methodology was used applying quantitative techniques. The method consisted of participant observation with registers on a filed diary associated to the application of a questionnaire on 18 participants from the nursing team of a Public Hospital in Rio de Janeiro. The study indicates that the basic needs of the nursing team are compromised, especially safety and physiological needs, which are the most primary. The compromising of the subjects' primary needs implies health hazards and reduced work performance in hospital settings.
\end{abstract}

\section{RESUMEN}

El cuidado está asociado a las acciones de enfermería considerando un conjunto de necesidades del equipo de enfermería. Los objetivos fueron: caracterizar situaciones de (in)satisfacción del equipo de enfermería en el trabajo y analizar sus implicaciones. Se adoptó la teoría de las necesidades humanas básicas de Maslow para comprender factores motivacionales, utilizándose una metodología cualitativa con aplicación de técnicas cuantitativas. El método se compuso de observación participativa con registro diario de campo asociada a cuestionario, aplicándose en 18 miembros del equipo de enfermería de un hospital público de Rio de Janeiro. El estudio indica que las necesidades básicas del equipo de enfermería se encuentran comprometidas, principal y primordialmente las de seguridad y fisiológicas. EI compromiso de las necesidades primarias del sujeto cuidador implica un perjuicio para la salud y reducción del desempeño en el trabajo en escenarios hospitalarios.

\author{
DESCRIPTORS \\ Nursing, team \\ Job satisfaction \\ Occupational health
}

\author{
DESCRIPTORES \\ Grupo de enfermería \\ Satisfacción en el trabajo \\ Salud laboral
}

\footnotetext{
* Extraído da dissertação "A equipe de enfermagem e suas (in)satisfações no trabalho em cenários hospitalares", Escola de Enfermagem Anna Nery da Universidade Federal do Rio de Janeiro, 2006. ' 1 Enfermeira. Mestre em Enfermagem. Doutoranda do Programa de Pós-Graduação da Escola de Enfermagem Anna Nery da Universidade Federal do Rio de Janeiro. Professora do Curso de Graduação em Enfermagem da Universidade Estácio de Sá. ladeiavr@yahoo.com.br ${ }^{2}$ Doutora em Enfermagem. Professora Adjunta do Departamento de Enfermagem Médico Cirúrgica da Escola de Enfermagem Anna Nery da Universidade Federal do Rio de Janeiro. Pesquisadora do Núcleo de Pesquisa em Enfermagem Hospitalar e do CNPq. isaura70porto@gmail.com

$\begin{aligned} \text { Rev Esc Enferm USP } & \text { Recebido: 18/06/2009 } \\ \text { 2011; 45(2):334-41 } & \text { Aprovado: 29/07/2010 }\end{aligned}$
}




\section{INTRODUÇÃO}

No decorrer de sua história, a Enfermagem vem acumulando conhecimentos empíricos e seus profissionais têm desenvolvido suas atividades baseadas em normas e rotinas repetidas, muitas vezes sem reflexão sobre a sua atuação, mesmo com as modificações na postura da clientela, da organização das instituições e do avanço tecnológico na área da saúde. Como conseqüência desse enfoque, de maneira geral, os pacientes têm recebido uma assistência de enfermagem mecanizada e os profissionais de enfermagem deixam de alcançar suas necessidades. Esta situação contribui para um contexto de insatisfações, o que leva a uma desvalorização do cuidado. Assim, esta pesquisa buscou as (in)satisfações dos integrantes da equipe de enfermagem e suas implicações sobre as atividades assistenciais oferecidas.

As expectativas, os desejos e as necessidades emergem nos integrantes da equipe de enfermagem contribuindo para a criação, interelação e desenvolvimento do ambiente de trabalho. As necessidades e expectativas não atendidas dos integrantes da equipe de enfermagem passam a refletir também no cuidado de enfermagem. Para compreender e pesquisar no cenário de saúde é preciso considerar que a Enfermagem é uma ciência em construção. Até bem recentemente a profissão vinha adotando um critério de cientificidade derivado de um senso comum construído a partir da reflexividade originada nos grupos da Enfermagem. Aos poucos, ela vem assumindo uma posição de ciência stricto sensu, através da adoção de uma postura crítica baseada em cânones científicos estabelecidos pela pesquisa tradicionalmente desenvolvida em outras áreas. Assim, sua produção cada vez mais, fundamenta-se em pesquisas que fortalecem a sua prática ${ }^{(1)}$.

Nesta perspectiva, para uma compreensão mais abrangente da Enfermagem torna necessária a utilização de uma teoria prática de cuidar baseada em dimensões que envolvam o corpo de quem cuida e é cuidado, como força e poder indissociável à vida, e, portanto, de bem-estar ${ }^{(2)}$. Partindo destas reflexões, é impossível obter a satisfação dos clientes externos (pacientes), sem que os clientes internos (integrantes da equipe) estejam igualmente satisfeitos com as condições, local e a dinâmica do trabalho.

Entre os aspectos que levam à satisfação dos integrantes da equipe de saúde, é vital entender que sua satisfação num ambiente de trabalho não decorre somente e basicamente do fator salário. Ele apenas contribui juntamente aos demais benefícios: salário indireto - ticket refeição, plano de saúde e odontológico, vale transporte e aspectos correlacionados às relações no trabalho - tratamento solidário, gentil, equilíbrio positivo e justo, liberdade de expressão, satisfação pelo que se faz, atenção, elogio e consideração como ingredientes indispensáveis na busca de lealdade, qualidade e produtividade no ato de cuidar $^{(3)}$.

O cuidar deve ser entendido como a realização de ações na Enfermagem, considerando-se inevitável a satisfação de um conjunto de necessidades que quando atendidas possibilitam maior envolvimento dos integrantes da equipe de enfermagem em suas atividades com cuidados de qualidade e melhor desempenho no trabalho. Então, a excelência na assistência de enfermagem está intimamente relacionada aos estados fisiológicos, de segurança e psicológicos dos integrantes desta equipe que cuida, pois eles fazem parte de um processo dinâmico indissociável dentro do trabalho.

\section{OBJETIVOS}

Este estudo leva a repensar a assistência a partir de conceitos, valores, relações e estruturas relacionadas com o cuidado. Assim, o objeto de estudo é as (in)satisfações dos integrantes da equipe de enfermagem no trabalho e suas consequências sobre as atividades oferecidas. Em seu desenvolvimento, o estudo estabeleceu os seguintes objetivos: a) Caracterizar situações de (in)satisfações dos integrantes da equipe de enfermagem no trabalho; b) Analisar as implicações das (in)satisfações dos integrantes da equipe de enfermagem no trabalho.

Sua contribuição encontra-se na compreensão mais aprofundada sobre o que move os integrantes da equipe de enfermagem, em sua prática cotidiana dos cuidados de enfermagem, em diversos cenários hospitalares. Essa compreensão traz uma contribuição para a assistência, o ensino, a pesquisa e a saúde do trabalhador de enfermagem. Essa visão leva a considerar uma nova proposta de cuidar, tendo como foco o integrante da equipe, para desenvolver uma assistência de excelência, na medida em que estando a equipe mais integrada, autônoma, atuante e satisfeita, ela constrói um ambiente de trabalho mais saudável, feliz e eficaz, capaz de tornar possível, no dia-a-dia, a (re)construção de um cuidado estruturado, planejado e sólido e caracterizado pela confiança e credibilidade.

\section{FUNDAMENTAÇÃO TEÓRICA}

Partindo da premissa de que o homem é motivado pelo desejo de satisfazer muitas necessidades, Abraham $\mathrm{H}$. Maslowe estruturou sua Teoria da motivação humana considerando uma hierarquia das necessidades humanas básicas $^{(4)}$. Esta teoria parte do princípio de que todo ser humano tem necessidades comuns que motivam seu comportamento no sentido de satisfazê-las, de acordo com níveis hierárquicos. A classificação hierárquica das necessidades é apresentada em cinco níveis, a saber: 
a) Necessidades básicas ou fisiológicas: aquelas diretamente relacionadas à existência e a sobrevivência do ser humano, tais como: alimento, água, vestuário, sexo e saneamento;

b) Necessidades de segurança: as necessidades relacionadas à proteção individual contra perigos e ameaças tais como: saúde, trabalho, seguro, previdência social e ordem social;

c) Necessidades de amor e/ou sociais: estão relacionadas à vida em sociedade, englobando as necessidades de convívio, amizade, respeito, amor, lazer e participação, referindo-se à necessidade de afeto das pessoas, tais como amigos, noiva, esposa e filhos;

d) Necessidades de estima: guardam relação com a auto-satisfação, tais como: independência, apreciação, dignidade, reconhecimento, igualdade subjetiva, respeito e oportunidades, referindo-se à uma auto-avaliação estável, bem como, uma auto-estima alta conduzindo a sentimentos de autoconfiança, valor, força, capacidade, suficiência e utilidade ao mundo;

e) Necessidades de auto-realização: expressam o mais alto nível das necessidades estando diretamente relacionadas à realização integral do indivíduo. Neste grupo destacam-se a utilização plena de suas potencialidades, sua capacidade e existência de ideologias. Além das cinco necessidades acima, Maslow acrescentou à sua teoria, o desejo de todo ser humano de saber e conhecer, ou seja, a necessidade natural do ser humano de buscar o sentido das coisas, de forma a organizar sua compreensão sobre o mundo em que vive. São as necessidades cognitivas, tais como: desejo de saber, compreender, sistematizar, organizar, analisar e procurar relações e sentidos. Estas necessidades viriam antes da auto-realização. Destacam-se ainda a necessidade de ajudar os outros a se auto-desenvolverem e a realizarem seu potencial, as necessidades transcendentes, que viriam depois da auto-realização.

O comportamento é motivado pelas necessidades fundamentais. Assim, ele destaca que las necesidades básicas se ordenan por si mismas en una jerarquía perfectamente definitiva, sobre la base del principio de potencia relativa ${ }^{(4)}$. Tais necessidades são baseadas na sua classificação em dois estados: o estado de deficiência ou inferiores ou o estado de crescimento ou superiores.

As necessidades de deficiência ou inferiores são as fisiológicas, de segurança, de afeto, auto-estima e cognitivas sendo que sua ausência indica insatisfações. Já as necessidades de crescimento ou superiores são aquelas relacionadas ao auto-desenvolvimento e a auto-realização dos seres humanos. Geram fatores motivacionais que estimulam as metas de responsabilidades, crescimento e inovação levando o ser humano a atingir a satisfação. Assim, quando estas necessidades estão satisfeitas significa que o indivíduo alcançou maior eficiência biológica, maior longevidade, menos enfermidades ${ }^{(5)}$.
Neste contexto, a pesquisa relaciona a Teoria da Motivação com a Enfermagem, buscando compreendê-la como uma profissão a serviço do ser humano e da dinâmica que envolve quem cuida e é cuidado. Na profissão estão implícitas as relações humanas e as implicações que definem sua prática e tudo a sua volta ${ }^{(6)}$.

\section{MÉTODO}

Este estudo adotou uma abordagem qualitativa com adoção de técnicas quantitativas. Neste sentido, a pesquisa qualitativa trabalha com o universo de significados, motivos, aspirações, crenças, valores e atitudes, o que corresponde a um espaço mais profundo das relações, dos processos e dos fenômenos ${ }^{(7)}$. O uso de técnicas quantitativas aplicadas a este estudo justificou-se pela possibilidade de ampliar e enriquecer a análise dos dados. A aplicação das técnicas quantitativas tornou possível apontar para as prováveis causas das (in)satisfações dos integrantes da equipe de enfermagem, assim como descrever em detalhes o padrão de ocorrência dos eventos observados. A combinação de métodos qualitativos com técnicas quantitativos utilizados configura-se numa estratégia conhecida como triangulação simultânea, no qual os dois métodos são usados ao mesmo tempo, para fornecer informações complementares acerca do estudo proposto(8).

Foi aprovado pelo Comitê de Ética e Pesquisa (CEP) da EEAN/HESFA na reunião de 30 de agosto de 2005, número do protocolo 047/05. Os cenários selecionados para o desenvolvimento do estudo foram enfermarias de um hospital público especializado em cardiologia, de grande porte, com 203 leitos instalados (168 em funcionamento). O setor no qual foram selecionadas as enfermarias agrupa clientes portadores de doenças coronariopatas, nos períodos pré e pós-operatório de angioplastia e cirurgia de revascularização miocárdica. Este setor tem 29 leitos (cada duas unidades de clientes são independentes tendo um banheiro comum).

Como critério de inclusão para participar da pesquisa, os participantes do estudo tinham que fazer parte da equipe de enfermagem, distribuídos em diferentes plantões da unidade hospitalar de período pré-operatório. Sendo assim foram envolvidos dezoito participantes, sendo seis enfermeiras (uma supervisora, duas diaristas e três plantonistas do serviço noturno), quatro técnicas (três plantonistas do serviço diurno e uma plantonista do serviço noturno) e oito auxiliares de enfermagem (uma diarista, três plantonistas do serviço diurno e quatro do serviço noturno), todos do sexo feminino, com idades variando de 21 a 58 anos, tempo de exercício profissional de nove meses a 31 anos e tempo de trabalho na instituição entre nove meses e 25 anos. A preocupação em ampliar a participação de diferentes categorias da Enfermagem ofereceu múltiplas dimensões e dados mais representativos sobre o contexto do cuidado em âmbito social. Isso implicou em considerar como sujeitos de estudo: gente, em determinada condição social, pertencente a determinado grupo soci- 
al ou classe com suas crenças, valores e significados amplos $^{(9)}$. Mesmo procurando a diversificação na participação dos integrantes da equipe de enfermagem, os resultados desta pesquisa somente são válidos para o setor no qual foram coletados os dados.

As técnicas de pesquisa adotadas como instrumentos para coleta de dados foram: a) a observação participante como principal instrumento; b) o questionário, como técnica complementar à observação participante. A coleta de dados foi realizada entre 08 e 14/09/2005, nos turnos da manhã, tarde e noite, para cobrir todas as equipes da unidade hospitalar de período pré-operatório. Os dados foram registrados em diário de campo com anotações de cunho descritivo e reflexivo (sobre ambiente, sujeitos, situações, datas, horários que aconteceram nas observações) e serviram de documentação para classificar e esclarecer algumas das situações práticas ocorridas no campo da pesquisa. Em um segundo momento, o questionário foi um instrumento utilizado a partir da observação participante, como uma técnica complementar com a finalidade de buscar no próprio campo e junto aos participantes, o que era necessário para responder as dúvidas levantadas pela pesquisa.

A análise foi realizada em três etapas utilizando instrumentos qualitativos e quantitativos de maneira seqüencial para compreender melhor e amplamente os resultados. $\mathrm{Na}$ primeira etapa, a observação participante descrita no diário de campo forneceu dados qualitativos, por meio dos quais foi possível categorizá-los quanto às necessidades humanas básicas envolvidas correlacionando-as as categorias de enfermagem (enfermeiros, técnicos e auxiliares). Esta técnica seguiu o modelo por caixa preconizado na análise de conteúdo categorial ${ }^{(10)}$, para classificar e categorizar os dados coletados.

Após identificadas as necessidades dos participantes da pesquisa, a segunda etapa da pesquisa foi desenvolvida consistindo na inclusão daqueles aspectos frente aos quais os integrantes da equipe de enfermagem mostraram incômodo em seu ambiente de trabalho. Estas respostas fo- ram apresentadas na análise em conjunto com os resultados derivados da observação, com a finalidade de confrontar ambos os resultados para identificar as confirmações e contradições existentes entre o que foi observado e o que foi dito. Na terceira e última etapa os resultados qualitativos e quantitativos confrontados foram relacionados à Teoria da Motivação Humana.

\section{RESULTADOS}

Os dados foram apresentados conforme o nível hierárquico das necessidades da Teoria da Motivação Humana consideradas como categorias analíticas por trazerem em si um substrato teórico. Foram analisadas as necessidades fisiológicas, de segurança, sociais, de auto-estima, autorealização e transcendentes.

As situações observadas e registradas no diário de campo foram classificadas em insatisfatórias e satisfatórias, perfazendo um total de cento e quatro registros. Deste total, noventa situações foram caracterizadas como insatisfatórias e quatorze como satisfatórias. Das necessidades fisiológicas representadas, doze registros foram classificados como insatisfatórios e 3 como satisfatórios; das necessidades de segurança, trinta e uma foram classificadas como insatisfatórias e 1 delas como satisfatória; das necessidades sociais encontradas, vinte e oito situações foram consideradas como insatisfatórias e 1 delas como satisfatória; as necessidades de auto-estima apresentaram-se com quinze situações insatisfatórias e 8 como satisfatórias; as necessidades de auto-realização tiveram o menor número de registros, 4 consideradas como situações insatisfatórias.

Os outros dados derivados do questionário abordaram as percepções insatisfatórias, parcialmente satisfatórias e satisfatórias dos dezoito integrantes da equipe de enfermagem em relação às necessidades levantadas (fisiológicas, segurança, social, auto-estima, auto-realização e transcendentes), cujo resultado é apresentando na Figura 1.

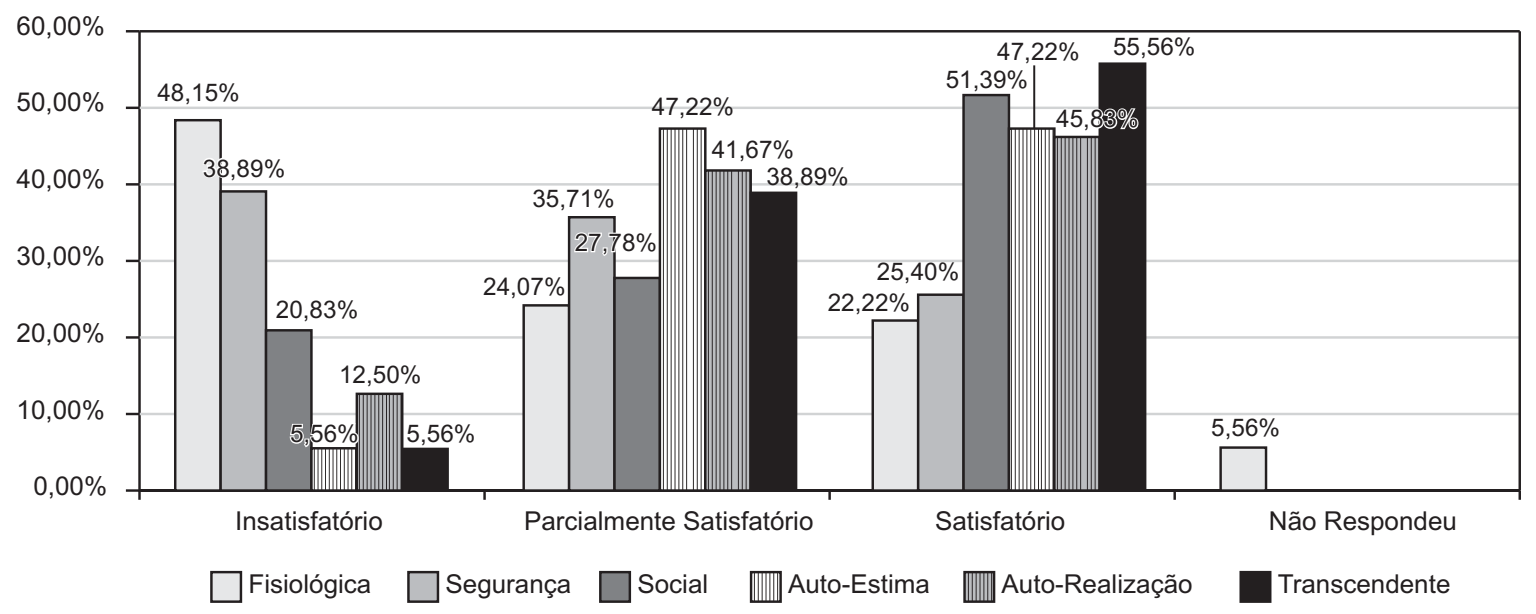

Figura 1 - Necessidades humanas básicas dos integrantes da equipe de enfermagem - Rio de Janeiro - 2006 
As necessidades fisiológicas e de segurança apresentaram os maiores índices de insatisfação. Já as necessidades de auto-estima e auto-realização apareceram em plano secundário, como parcialmente satisfatórias. E a necessidade transcendente apresenta um alto índice de satisfação. As insatisfações encontradas estão relacionadas com vários aspectos levantados pelos participantes da pesquisa detalhados a seguir:

\section{Necessidades fisiológicas}

- Hidratação: os integrantes da equipe de enfermagem rateavam o valor referente à água que compravam para beber, por acreditarem que a água sem filtragem existente nos bebedouros do hospital era imprópria para o consumo;

- Alimentação inadequada, falta de louça e variedade no cardápio: os participantes destacaram que a cozinha é mal higienizada, o refeitório apresenta-se sem ventilação, a alimentação é gordurosa, indigesta e faltam opções no cardápio para consumo. Reforçando estes destaques, muitas vezes eles tinham que esperar a louça ser lavada para se alimentarem, o que atrasava o retorno ao trabalho. Acrescentam que, algumas vezes, a quantidade da comida era insuficiente. Ainda pode-se ressaltar que era proibida a reserva de almoço pelos integrantes, mesmo que estivessem envolvidos em uma emergência. Esta é uma questão bastante delicada, pois um funcionário em uma emergência pode estar impossibilitado de descer para o refeitório no horário estabelecido ficando sem almoço;

- Quanto ao descanso: o local de repouso era distante, pequeno, incômodo, desconfortável, sem banheiro e desorganizado. Os integrantes da equipe, além de sujeitados a cubículos apertados para guardarem seus pertences e fazerem seu descanso, ainda precisavam deslocar-se até o posto de enfermagem para chegar ao banheiro. Eles destacaram também, que o tempo de descanso era curto no serviço noturno, pois o déficit de funcionários muitas vezes não permite um revezamento. $\mathrm{E}$, existia a falta de tempo para descanso no serviço diurno, tudo isto ampliado pela sobrecarga de trabalho.

\section{Necessidades de segurança}

- Materiais, medicações e estrutura física: apesar destes apresentarem-se pouco abordados pelos participantes, estiveram presentes. Eles afirmam que, na maioria das vezes, não faltam medicamentos no setor; mas, quando faltam são comprados pela instituição. A questão mais relevante envolve os materiais usados na assistência, as camas utilizadas pelos pacientes em estado inadequado, por estarem enferrujadas, com manivelas emperradas e com ausência de grades. Estes aspectos dificultam a assistência de enfermagem, principalmente durante os cuidados a clientes idosos. Outras questões relacionadas com a estrutura física do hospital referiam-se a enfermarias pequenas para realizar procedimentos, principalmente em caso de parada cárdio-respi- ratória do cliente; a localização inadequada do posto de enfermagem, por estar descentralizado, isolado e distante das últimas enfermarias;

- Salário, carga, déficit de funcionários e lazer: são aspectos significativos no que refere a insatisfação dos integrantes da equipe de enfermagem. Eles apontaram a defasagem do salário há dez anos e relataram que a sobrecarga de trabalho só aumenta devido à falta de funcionários dos setores. A distribuição de funcionários é de 2 auxiliares ou técnicos de enfermagem para vinte e nove clientes com complexidades variáveis em suas condições clínicas. Além de oferecerem uma assistência deficiente para os clientes, o absenteísmo apresentado e as licenças crescentes podem ser atribuídos a esta deficiência de pessoal. Acentuando este quadro, a instituição não oferece nenhuma atividade de lazer ou anti-estresse para seus funcionários.

\section{Necessidades sociais}

- Participação e relacionamento: algumas situações foram relacionadas ao convívio social da equipe de enfermagem. Entre eles se destacaram a ausência de participação dos funcionários nos processos decisórios da instituição, a falta de comunicação com a chefia de enfermagem e equipe médica e o relacionamento conflituoso com os acompanhantes dos clientes internados.

\section{Necessidades de auto-estima}

- Autonomia e reconhecimento: outras dificuldades da equipe de enfermagem no trabalho foram apontadas pelos seus integrantes como falta de reconhecimento e elogios da chefia de enfermagem e dos clientes em relação ao cuidado que oferecem. Destacaram também, a falta de liberdade de expressão por parte de alguns funcionários pertencentes à cooperativa, pela instabilidade decorrente da contratação (sem concurso), o que facilita sua demissão em qualquer momento.

\section{Necessidades de auto-realização}

- Em relação aos sentimentos voltados à profissão, os integrantes da equipe relataram que era desvalorizada por eles mesmos e pela instituição. Destacaram ainda que inexiste incentivo institucional para aprimorar o crescimento e desenvolvimento da equipe de enfermagem. Quanto ao seu desempenho, a falta de treinamentos e o déficit de funcionários não permitiam uma melhor e maior dedicação aos clientes.

As satisfações encontradas em relação às necessidades foram:

\section{Necessidades fisiológicas}

- Descanso proporcionado pela poltrona localizada no posto de enfermagem considerada confortável e o conforto derivado da roupa utilizada no trabalho. 


\section{Necessidades segurança}

- A estabilidade no emprego para concursados.

\section{Necessidades sociais}

- A boa comunicação dos médicos plantonistas com as enfermeiras do serviço noturno, o bom relacionamento com alguns colegas de trabalho e pacientes e a ajuda dos colegas nas atividades realizadas.

\section{Necessidade de transcendência}

- Nos dados de ambos os instrumentos deste estudo, esta necessidade apareceu com um predomínio de satisfação. Porém, o aspecto apontado pelos integrantes da equipe relacionou-se a cumplicidade entre colegas e não ao aprofundamento característico desta necessidade. Isto indica que esta necessidade precisa ser melhor explorada em outras pesquisas.

\section{DISCUSSÃO}

As necessidades apresentadas na Figura 1 mostram que as insatisfações dos integrantes da equipe de enfermagem concentram-se nas necessidades fisiológicas e de segurança. Estas necessidades encontradas nos resultados do diário de campo e questionário apontam para a pessoa com uma carência de evolução relativa às necessidades básicas. Os integrantes da equipe de enfermagem ainda estão tentando suprir e consolidar o atendimento de suas necessidades mais primárias, o que envolve o acesso à água, alimentação, ao descanso e o provimento de número suficiente de funcionários, para somente depois estarem aptos a atingir o máximo de suas potencialidades relativas às necessidades superiores de auto-estima, auto-realização e transcendentes.

Sendo assim, as insatisfações da equipe de enfermagem podem interferir no alcance de outras necessidades (autonomia, reconhecimento e crescimento profissional) comprometendo sua plena satisfação e desenvolvimento adequado no trabalho. Neste sentido,

a enfermeira [e os demais integrantes da equipe] utiliza[m] mecanismos e estratégias de resistência não só individuais e informais (omissão, adesão e inovação), como também formais e coletivos (formação de alianças, denúncias e manifestações), em contraposição às relações de dominação existentes em seu trabalho ${ }^{(11)}$.

O desânimo, desinteresse e estresse dos profissionais no e com o trabalho podem gerar conseqüências prejudiciais em sua saúde e seu trabalho. Diante disto, para garantir uma boa assistência de enfermagem é necessário que os integrantes da equipe de enfermagem estejam mais satisfeitos, para que seu desempenho ao cuidar dos clientes possa estar plenamente desenvolvido. Neste contexto, a

questão da satisfação no trabalho parece ser tão complexa como o próprio homem. Esta complexidade decorre dos múltiplos aspectos que influenciam o comportamento do homem (necessidades, expectativas, experiências anteriores, valores, fatores culturais), bem como dos fatores ligados à organização (regras, tipo de trabalho, conceito de empresa) e dos seus integrantes (relacionamento interpessoal, aceitação grupal, valorização, dentre outros) ${ }^{(12)}$.

Depois destes resultados tornou-se mais fácil a compreensão de que, quando um indivíduo ainda está buscando alcançar suas necessidades primárias, não consegue almejar seu desenvolvimento e crescimento profissional, que são aspirações relacionadas com suas necessidades superiores. Em outro estudo foi relatado que quando a necessidade de segurança encontra-se como a mais afetada, por não encontrar condições de trabalho favoráveis ao desempenho de suas atividades, conseqüentemente afetará sua necessidade de auto-realização ${ }^{(13)}$. As necessidades superiores somente são ambicionadas e buscadas, quando as mais básicas estão satisfeitas. Confirmando esta análise, uma pessoa só poderá dar ao outro algo que possua ${ }^{(3)}$. E, os integrantes da equipe de enfermagem ainda precisam entender e satisfazer suas necessidades.

Procurar conhecer as melhores técnicas para aprimorar o cuidado ao paciente torna-se algo sem significado, quando não se tem acesso a água filtrada para beber e uma compatibilidade entre salário e despesas. É provável que a pessoa deixe de ser bem sucedida no trabalho, sem antes atender as necessidades básicas para sua sobrevivência. A enfermeira e os demais integrantes da equipe com sede, fome, cansaço, sono, tristeza, insatisfação e sem reconhecimento não conseguem estar plenamente atentos a outro ser humano. Eles precisam atender as suas próprias necessidades para somente depois perceber/suprir as necessidades do outro.

É justamente na influência decorrente de necessidades insatisfeitas que se fundamentam as possíveis conseqüências para o cuidado, pois a enfermeira e os demais integrantes da equipe tenderão a reproduzir suas insatisfações na relação do cuidado com o outro, podendo comprometê-lo. Ao lado disto o enfermeiro e a instituição hospitalar devem reconhecer os estressores que estão presentes no trabalho e procurar mecanismos e estratégias de enfrentamento individual e grupal para diminuir a ocorrência de estresse profissional ${ }^{(14)}$.

O envolvimento e participação dos trabalhadores com o processo de trabalho e a reestruturação produtiva em constante mudança podem e devem influenciar-se, evitando que a nova relação traga riscos para a saúde dos trabalhadores ${ }^{(15)}$.

Neste caso, a implicação para o cuidado advém do tipo de motivação e insatisfação no trabalho que podem influenciar numa assistência de melhor qualidade.

Em virtude disto, vale sugerir que as organizações avaliem os fatores de insatisfação no trabalho do enfermeiro para que este profissional possa fazer uma assistência de meIhor qualidade, o que conseqüentemente contribuirá para o sucesso da empresa ${ }^{(13)}$. 
As condições e relações no trabalho podem afetar diretamente a satisfação, a saúde dos integrantes da equipe de enfermagem e a qualidade dos serviços.

\begin{abstract}
Podemos considerar que os profissionais mais insatisfeitos com o trabalho encontram-se submetidos a longas jornadas de trabalho, a alta exposição a riscos químicos e físicos, a falta de reconhecimento profissional, entre outros estressores ocupacionais inerentes à profissão, que afetam diretamente sua satisfação laboral e conseqüentemente a qualidade dos serviços ${ }^{(16)}$.
\end{abstract}

\section{CONCLUSÃO}

Esta investigação procurou desvendar as (in)satisfações dos integrantes da equipe de enfermagem no trabalho evidenciadas através de suas necessidades e suas influências sobre o cuidados de enfermagem. As implicações de seus resultados para o cuidado de enfermagem configuram-se como uma limitação do estudo, o que indica a realização de novas investigações, pois a relação necessidades - cuidado foi estabelecida predominantemente com base na literatura.

As insatisfações mais encontradas estiveram relacionadas com vários aspectos levantados pelos participantes da pesquisa, tais como: hidratação, alimentação, descanso, salário, carga e déficit de funcionários. As necessidades fisiológicas e de segurança apresentaram os maiores índices de insatisfação. Já as necessidades de auto-estima e auto-realização apareceram em plano secundário, como parcialmente satisfatórias. As necessidades de transcendência ainda precisam ser mais exploradas em futuras investigações.

Diante da descrição destes aspectos envolvidos em questões conflituosas e insatisfatórias em seu dia-a-dia, a equipe de enfermagem parece utilizar mecanismos e estratégias de resistência em relação ao trabalho, muitas vezes sem perceber tal posição ou apenas conformando-se às situações que surgem. Para minimizar esta situação torna-se necessário que o grupo realize discussões sobre suas insatisfações indicando para a gerência suas possíveis implicações para o cuidado realizado.

Sem dúvida, esta pesquisa gerou avanços para melhor compreender a prática da enfermagem, ao direcionar a atenção para o cuidado a partir do referencial de quem cuida, com uma nova proposta de estudo para Enfermagem. Outro avanço ocorreu pelo envolvimento da insatisfação dos integrantes da equipe de enfermagem com a dinâmica e as interfaces da Teoria da Motivação Humana (Maslow), que pôde expor parte das estratégias de defesa utilizadas no trabalho pela equipe de enfermagem.

Esta é uma pesquisa que descortina um novo e vasto campo de estudos para a Enfermagem Hospitalar, mas também avança para além dela, apontando para a saúde do trabalhador, o gerenciamento e a educação. Os aspectos relevantes dos resultados desta pesquisa vinculam-se ao alcance das necessidades dos integrantes da equipe de enfermagem e suas influências no o cuidado. Neste sentido os resultados também apontam para a saúde dos trabalhadores de enfermagem e a responsabilidade dos dirigentes, gerentes e coordenadores institucionais em relação a minimização dos efeitos nocivos do trabalho sobre os cuidadores de enfermagem em ambientes hospitalares, pois as insatisfações encontradas não foram apenas de cunho social ou pessoal, mas envolveram condições estruturais e ambientais. Cabe também às instituições de saúde atender algumas destas necessidades, pois todas elas comprometem o desempenho profissional e influenciam o desenvolvimento do cuidado em enfermagem.

\section{REFERÊNCIAS}

1. Porto I S. A Etnometodologia e suas interfaces com a pesquisa em enfermagem: saberes e práticas investigativas. In: Anais da Conferência Internacional do Brasil de Pesquisa Qualitativa 1; 2004; Taubaté, BR [CD ROM].

2. Figueiredo NMA, Santos I, Sobral VRS, Silva JR. Cuidar em saúde: lugar de um novo paradigma. Rev Bras Enferm. 1998;51(3):447-56.

3. Souza JG. Humanização hospitalar: bases e estratégia de integração e inter-relação na equipe de enfermagem [dissertação]. Rio de Janeiro: Escola de Enfermagem Anna Nery, Universidade Federal do Rio de Janeiro; 2000.

4. Maslow AH. Motivación y personalidad. Barcelona: Ed. Sagitário; 1954.
5. Vitoria Regis LFL. A equipe de enfermagem e suas (in)satisfações no trabalho em cenários hospitalares [dissertação]. Rio de Janeiro: Escola de Enfermagem Anna Nery, Universidade Federal do Rio de Janeiro; 2006.

6. Vitoria Regis LFL, Porto IS. A equipe de enfermagem e Maslow: as (in)satisfações no trabalho. Rev Bras Enferm. 2006;59(4):565-8.

7. Minayo MCS. Pesquisa social. 20ạ ed. Petrópolis: Vozes; 1994.

8. Marcus MT, Liehr PR. Abordagem de pesquisa qualitativa. In: Lobiondo-Wood G, Haber J. Pesquisa em enfermagem: métodos, avaliação crítica e utilização. 4ạ ed. Rio de Janeiro: Guanabara Koogan; 2001. p. 122-39.

9. Minayo MCS. O desafio do conhecimento: pesquisa qualitativa em saúde. 8a ed. São Paulo: Hucitec; 2004. 
10. Bardin L. Análise de conteúdo. Lisboa: Edições 70; 1979.

11. Nobrega-Therrien SM. Dominação e resistência no trabalho da enfermeira. Rev Bras Enferm. 2001;54(3):420-6.

12. Moura GMS. O estudo da satisfação no trabalho e do clima organizacional como fatores contributivos para o ser saudável no trabalho da enfermagem. Texto Contexto Enferm. 1992;1(2):167-79.

13. Batista AAV, Vieira MJ, Cardoso NCS, Carvalho GRP. Fatores de motivação e insatisfação no trabalho do enfermeiro. Rev Esc Enferm USP. 2005;39(1)85-91.
14. Guerrer FJL, Bianchi ERF. Caracterização do estresse nos enfermeiros de unidades de terapia intensiva. Rev Esc Enferm USP. 2008;42(2):355-62.

15. Neves MA. Trabalho e gênero: mudanças, permanências e desafios. Campinas: ABEP; 2000.

16. Lima Júnior J, Alchieri JC, Maia EMC. Avaliação das condições de trabalho em Hospitais de Natal, Rio Grande do Norte, Brasil. Rev Esc Enferm USP. 2009;43(3):670-6. 